\title{
DISCOVERY OF A NEW PHOTOMETRIC SUB-CLASS OF FAINT AND FAST CLASSICAL NOVAE
}

\author{
M. M. Kasliwal ${ }^{1}$, S. B. Cenko ${ }^{2}$, S. R. Kulkarni ${ }^{1}$, E. O. OfeK ${ }^{1}$, R. Quimby ${ }^{1}$, and A. RaU ${ }^{3}$ \\ ${ }_{1}^{1}$ Astronomy Department, California Institute of Technology, 105-24 Pasadena, CA 91125, USA; mansi@astro.caltech.edu \\ 2 Department of Astronomy, University of California at Berkeley, Berkeley, CA 94720, USA \\ ${ }^{3}$ Max-Planck Institut fuer Extraterrestrische Physik, 85748 Garching, Germany \\ Received 2011 March 1; accepted 2011 April 22; published 2011 June 21
}

\begin{abstract}
We present photometric and spectroscopic follow-up of a sample of extragalactic novae discovered by the Palomar 60 inch telescope during a search for "Fast Transients In Nearest Galaxies" (P60-FasTING). Designed as a fast cadence ( 1 day) and deep $(g<21 \mathrm{mag})$ survey, P60-FasTING was particularly sensitive to short-lived and faint optical transients. The P60-FasTING nova sample includes 10 novae in M 31, 6 in M 81, 3 in M 82, 1 in NGC 2403, and 1 in NGC 891. This significantly expands the known sample of extragalactic novae beyond the Local Group, including the first discoveries in a starburst environment. Surprisingly, our photometry shows that this sample is quite inconsistent with the canonical maximum-magnitude-rate-of-decline (MMRD) relation for classical novae. Furthermore, the spectra of the P60-FasTING sample are indistinguishable from classical novae. We suggest that we have uncovered a sub-class of faint and fast classical novae in a new phase space in luminosity-timescale of optical transients. Thus, novae span two orders of magnitude in both luminosity and time. Perhaps the MMRD, which is characterized only by the white dwarf mass, was an oversimplification. Nova physics appears to be characterized by a relatively rich four-dimensional parameter space in white dwarf mass, temperature, composition, and accretion rate.
\end{abstract}

Key words: galaxies: individual (M81, M82, M31, NGC 2403, NGC 891) - galaxies: starburst - novae, cataclysmic variables - surveys - techniques: photometric - techniques: spectroscopic

Online-only material: color figures

\section{INTRODUCTION}

Since the discovery of classical novae, astronomers have pursued their use as standard candles to determine distances (see Hubble 1929). Zwicky (1936) first noticed some regularity in nova light curves and termed this the "life-luminosity" relation. Arp (1956) undertook a comprehensive search for novae in M31, discovering 30 novae in 290 nights, and found a clear relation: luminous novae evolve faster than less luminous novae. The modern name for this observation is the maximummagnitude-rate-of-decline (MMRD) relation.

The MMRD relation has attracted considerable theoretical attention (e.g., Livio 1992). The basic idea is that the relation is entirely due to the mass of the accreting white dwarf. The more massive the white dwarf, the higher the surface gravity, the higher the pressure at the base of envelope and stronger the thermonuclear runaway (TNR; and hence, higher the peak luminosity). Also, the more massive the white dwarf, the smaller the envelope mass to attain the critical pressure for TNR and, hence, the faster the decline.

In more recent times, della Valle \& Livio (1995) used a sample of novae in M31 and the Large Magellanic Cloud (LMC) to propose an arctangent relation between the peak luminosity and the rate of decline. Downes \& Duerbeck (2000) used a sample of Galactic novae to propose a linear relation between the same two parameters. Darnley et al. (2006) used a score of novae in M31 from the POINT AGAPE survey and claimed their observations were consistent with the della Valle \& Livio (1995) formulation of the MMRD.

In comparison to supernovae, classical novae are not very luminous. Hence, searches (e.g., Shafter \& Irby 2001; Ciardullo et al. 1987) have traditionally focussed only on the Milky Way and its nearest neighbors (Andromeda and the LMC). Hornoch et al. (2008) looked into archival data and found 49 nova candidates $^{4}$ in M 81 in the past 20 years-unfortunately, these candidates have neither light curves nor spectra. Ferrarese et al. (2003) undertook a search for novae using 24 orbits of the Hubble Space Telescope (HST) and found nine nova candidates in M49. Even with their sparsely sampled light curves for nine novae, they concluded that novae are not good standard candles. Another survey, CFHT-COVET ${ }^{5}$ (aimed at finding transients in the gap between novae and supernovae) found a dozen nova candidates in many galaxies in the Virgo supercluster, including some in the far outskirts of galaxies (M. M. Kasliwal et al. 2011, in preparation).

Here, we report on novae discovered in high cadence monitoring observations of a representative collection of galaxies with distance less than that of the Virgo cluster. The original motivation of this search, P60-FasTING, ${ }^{6}$ was to explore rapid transients (those which last less than a couple of nights) in the nearest galaxies. A strong spectroscopic follow-up effort was a part of P60-FasTING. The survey was capable of finding novae in the major galaxies out to $4 \mathrm{Mpc}$ : M 31 (Figure 1), the starburst M 82 (Figure 2), NGC 2403 (Figure 3), and M 81 (Figure 4). We present our sample of 21 transients, which although spectroscopically indistinguishable from classical novae, photometrically occupy a new region of phase space.

The paper is organized as follows: Section 2 describes the discovery, photometric, and spectroscopic follow-up observations of this nova sample; Section 3 describes the data analysis; Section 4 discusses the implications; and Section 5 presents our conclusion.

\footnotetext{
4 We use the term candidate where the light curve is very sparse and/or there is no spectroscopic confirmation.

5 Canada-France-Hawaii Telescope COma Virgo Exploration for Transients.

6 Palomar 60 inch Fast Transient In Nearest Galaxies.
} 


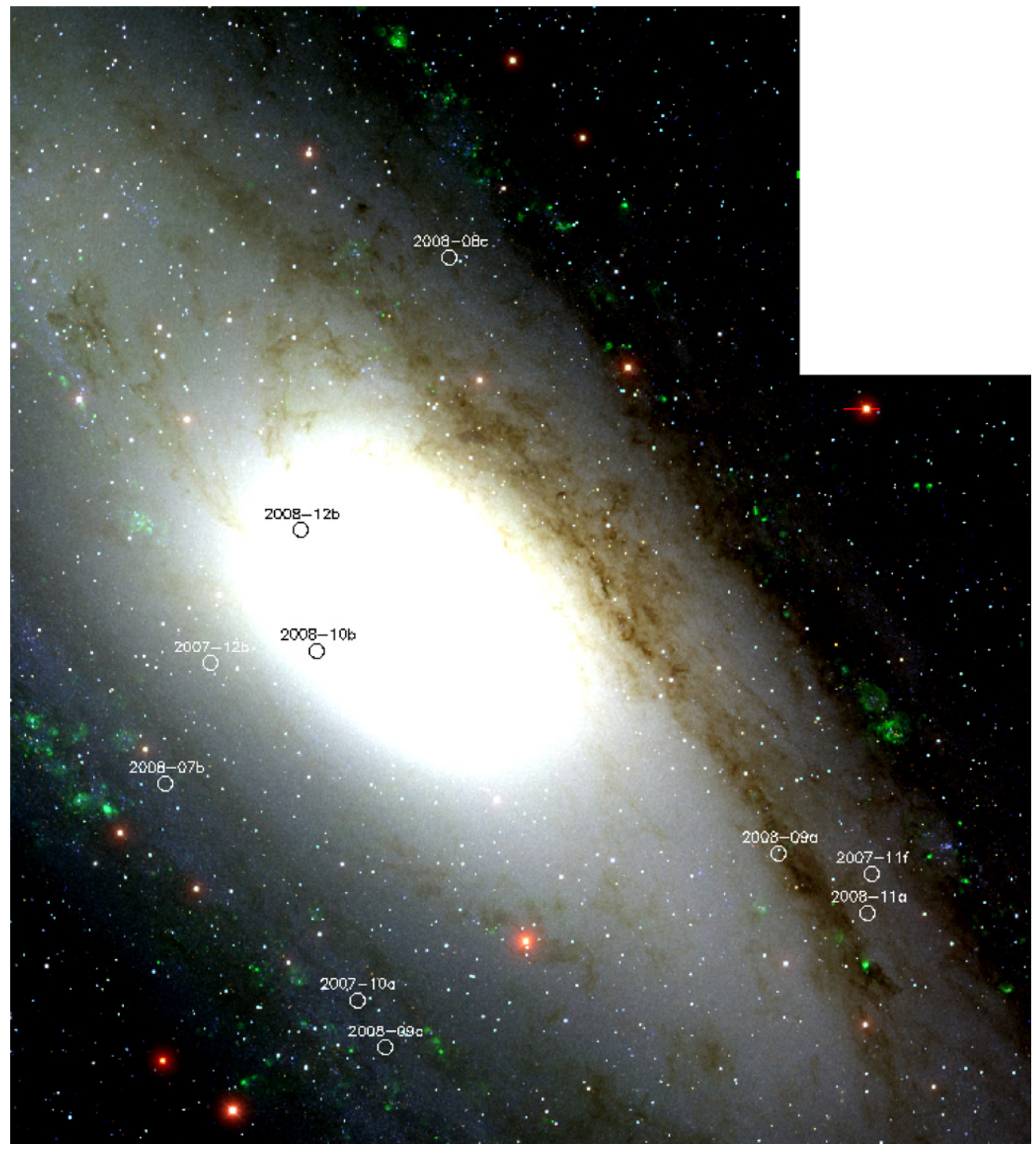

Figure 1. Location of 10 novae in M31. The background image is a mosaic based on Massey data. (A color version of this figure is available in the online journal.)

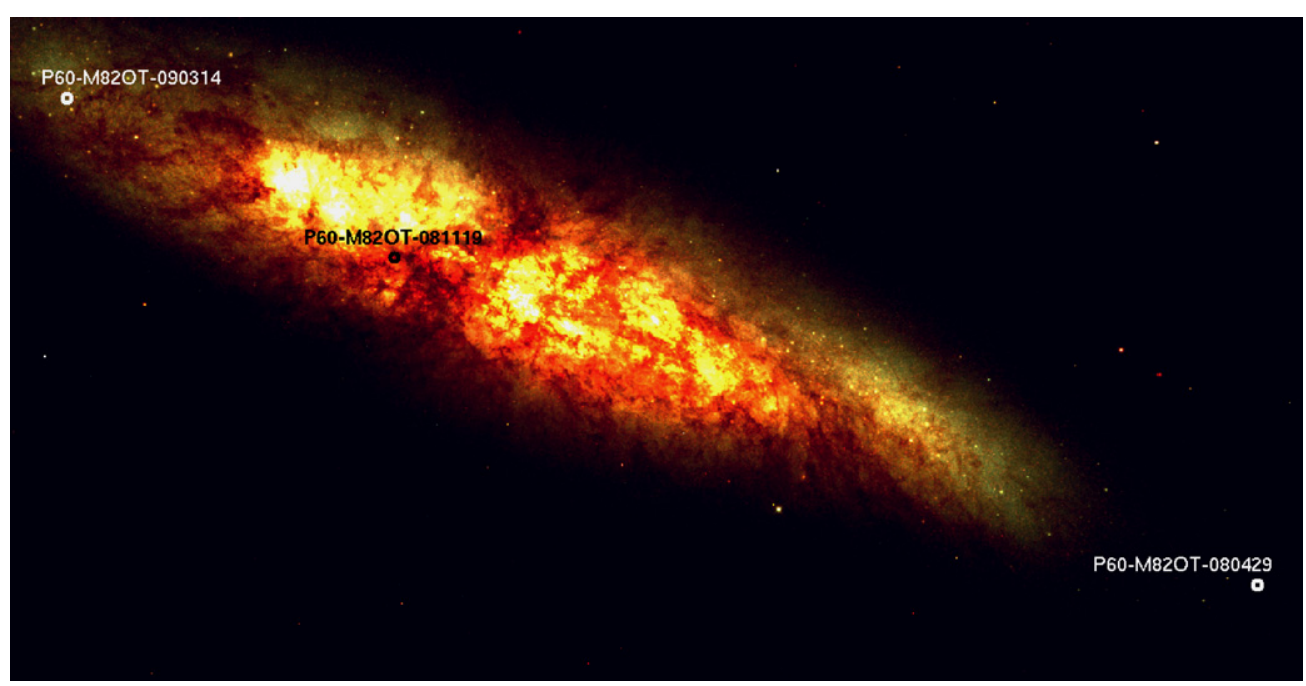

Figure 2. Location of three novae discovered by P60-FasTING in the starburst environment of M82. The background image is an HST/ACS mosaic. (A color version of this figure is available in the online journal.) 


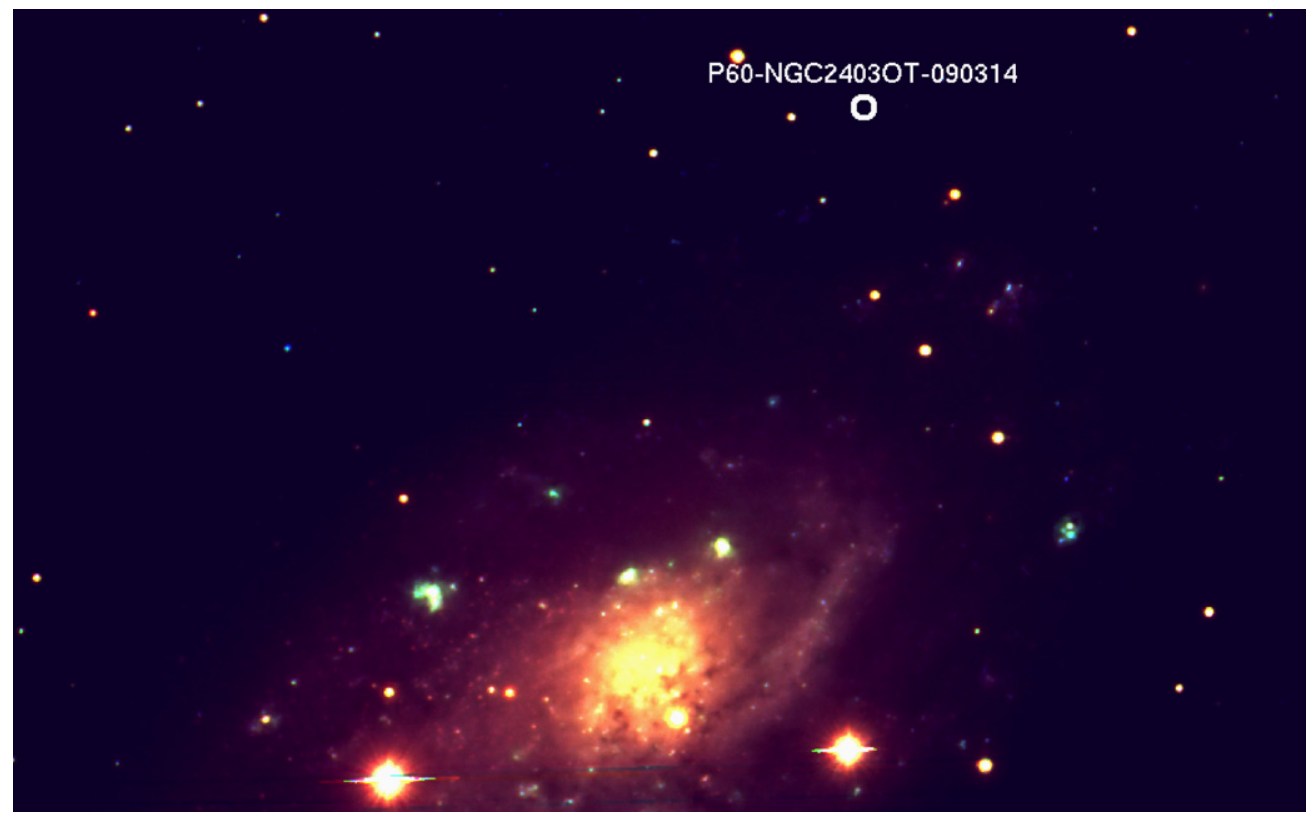

Figure 3. Location of one nova discovered by P60-FasTING in NGC 2403. The background image is a deep co-add of P60 data. (A color version of this figure is available in the online journal.)

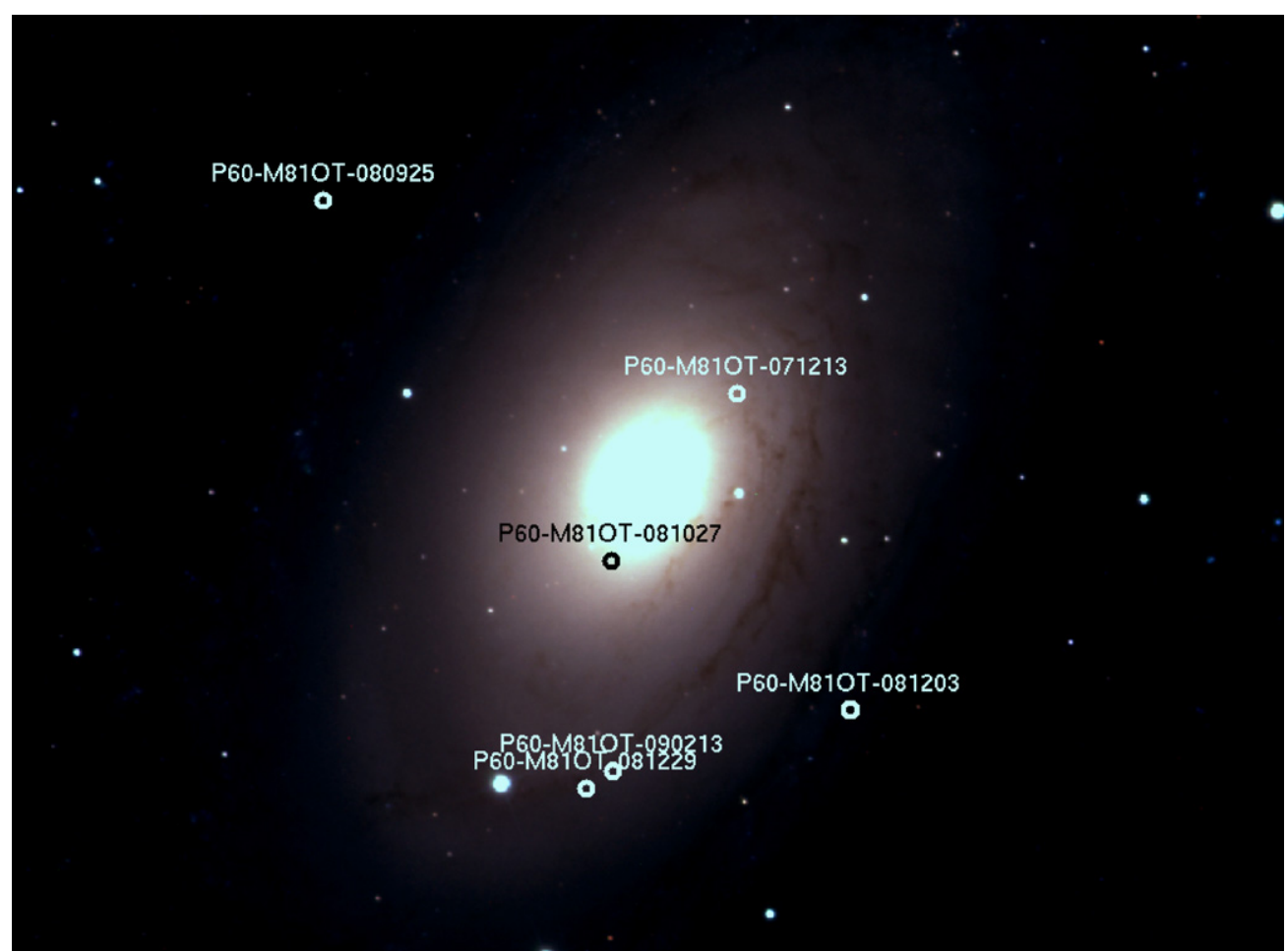

Figure 4. Location of six novae discovered by P60-FasTING in M81. The background image is a color mosaic using SDSS data. (A color version of this figure is available in the online journal.)

\section{OBSERVATIONS}

\subsection{Experiment Design}

P60-FasTING was designed with the specific goal of probing new phase space, particularly, fast transients with peak luminosity in the gap between novae and supernovae. The sample of galaxies included the brightest and nearest galaxies $(<20 \mathrm{Mpc}$, majority around $10 \mathrm{Mpc}$ ). The survey was undertaken in a single filter (primarily Gunn- $g$ and some Gunn- $i$ data just around full moon). The limiting magnitude was typically Gunn- $g<21$ and cadence was $<1$ day. The field of view of P60 is $13.5 \times 13.5$ and all galaxies except M31 were covered in a single pointing. For M31, five pointings were chosen to cover a larger fraction of the galaxy (Table 1).

A real-time data reduction and transient search pipeline was written and implemented in 2008 April. P60-FasTING ended in 2009 March. The search pipeline was written in python. A deep reference image was constructed by combining images from 
Table 1

P60-FasTING Pointing Centers in M31

\begin{tabular}{ll}
\hline \hline R.A. (J2000) & Decl. (J2000) \\
\hline $00: 42: 35.949$ & $+41: 02: 18.19$ \\
$00: 41: 26.856$ & $+41: 02: 24.84$ \\
$00: 43: 26.959$ & $+41: 15: 18.00$ \\
$00: 42: 54.236$ & $+41: 28: 18.33$ \\
$00: 44: 03.022$ & $+41: 28: 17.01$
\end{tabular}

several of the best seeing dark nights. Next, wcsremap was used to align every new image with the reference and hotpants was used to compute a convolution kernel prior to image subtraction (both codes supplied by A. Becker ${ }^{7}$ ). Although the image subtraction software was quite sophisticated in its convolution of the new image to match the reference prior to subtraction, we suffered from a large number of false positives. To distill the false subtraction residuals from the bona fide astrophysical sources, a variety of automatic filters were used (e.g., the shape characteristics of the point-spread function (PSF) of the candidate, how well it resembles the PSF characteristics of other stars in the image). However, the final step in the vetting process was done by human eye on candidate thumbnails every morning. Due to the myriad tradeoffs for maximum completeness and minimum contamination, the complex issue of quantifying the completeness of the nova sample is beyond the scope of this paper.

Our survey was sensitive to classical novae only in a handful of the nearest galaxies in our sample (distance, $d<4 \mathrm{Mpc}$ ). Classical novae discovered by P60-FasTING are summarized in Table 2. Some novae in M31 were announced by different groups before P60-FasTING's first detection (usually due to bad weather at Palomar); these are summarized in Table 3.

\footnotetext{
7 http://www.astro.washington.edu/users/becker/c_software.html
}

\subsection{Photometry}

The robotic Palomar 60 inch has a standard data reduction pipeline (Cenko et al. 2006). This pipeline performs basic detrending (flat fielding and bias subtraction) and computes an astrometric solution. In 2008 August, we added a new functionality: computation of a photometric solution. We used the Sloan Digital Sky Survey (SDSS) catalog where available, otherwise the NOMAD catalog. Note that where NOMAD was used (e.g., M31), the transformation from Johnson UBVRI magnitudes to SDSS ugriz magnitudes was done following Jordi et al. (2006).

To compute a light curve, we first measured the magnitude of the nova on the subtracted image. The subtracted image was scaled to the same flux level as the new image. Thus, we measured the magnitude of $\sim 150$ reference stars on the new image to compute a relative zero point with appropriate outlier rejection. Finally, we applied this relative zero point to the instrumental magnitude of the nova.

\subsection{Spectroscopy}

An integral part of P60-FasTING was follow-up spectroscopy to confirm and classify discovered candidate transients. Since we were looking for fast evolving phenomenon, we triggered our Target of Opportunity program on the Keck I and Palomar Hale telescopes soon after discovery. Sometimes due to bad weather or bright moon-phase ${ }^{8}$, neither of these was an option. We resorted to the queue-scheduled service-observed programs on the Gemini or HET telescopes. A log of spectroscopic observations can be found in Table 4.

\footnotetext{
8 Low-resolution spectrographs are usually available on both these telescopes only during the dark fortnight.
}

Table 2

Novae Discovered by P60-FasTING

\begin{tabular}{|c|c|c|c|c|c|c|}
\hline Nova & Host & Discovery Date & R.A. (J2000) & Decl. (J2000) & Offset from Host & Reference \\
\hline P60-NGC 2403-090314 & NGC 2403 & 2009 Mar 14.160 & $07: 36: 35.00$ & $+65: 40: 20.8$ & $101^{\prime \prime} .0 \mathrm{~W}, 252^{\prime \prime} .0 \mathrm{~N}$ & Kasliwal et al. (2009a) \\
\hline P60-M82OT-090314 & NGC 3034 & 2009 Mar 14.496 & $09: 56: 12.60$ & $+69: 41: 32.3$ & $104^{\prime \prime} .2 \mathrm{E}, 48^{\prime \prime} .2 \mathrm{~N}$ & $\ldots$ \\
\hline P60-M81OT-090213 & NGC 3031 & 2009 Feb 13.404 & $09: 55: 35.96$ & $+69: 01: 51.0$ & 15 "E, 124”S & Kasliwal et al. (2009b) \\
\hline P60-M31OT-081230 (2008-12b) & NGC 224 & 2008 Dec 30.207 & $00: 43: 05.03$ & $+41: 17: 52.3$ & 233".4E,103". $8 \mathrm{~N}$ & Kasliwal et al. (2009c) \\
\hline P60-M81OT-081229 & NGC 3031 & 2008 Dec 29.373 & $09: 55: 38.15$ & $+69: 01: 43.6$ & $26^{\prime \prime} 7 \mathrm{E}, 131^{\prime \prime} .4 \mathrm{~S}$ & Rau et al. (2009a) \\
\hline P60-M81OT-081203 & NGC 3031 & 2008 Dec 3.303 & $09: 55: 16.92$ & $+69: 02: 17.7$ & $87^{\prime \prime} .2 \mathrm{~W}, 97$ ". $4 \mathrm{~S}$ & Kasliwal et al. (2008a) \\
\hline P60-M82OT-081119 & NGC 3034 & 2008 Nov 19.536 & $09: 55: 58.39$ & $+69: 40: 56.2$ & $29.5 \mathrm{E}, 10 . .4 \mathrm{~N}$ & Kasliwal et al. (2008b) \\
\hline P60-M81OT-081027 & NGC 3031 & 2008 Oct 27.402 & $09: 55: 36.11$ & $+69: 03: 22.0$ & $15^{\prime \prime} 8 \mathrm{E}, 33^{\prime \prime} 1 \mathrm{~S}$ & Kasliwal et al. (2008g) \\
\hline P60-M81OT-080925 & NGC 3031 & 2008 Sep 25.49 & $09: 55: 59.35$ & $+69: 05: 57.1$ & $2 ! 35 \mathrm{E}, 2^{\prime} .03 \mathrm{~N}$ & Kasliwal et al. (2008i) \\
\hline P60-M31OT-080915 (2008-09c) & NGC 224 & 2008 Sep 15.36 & $00: 42: 51.42$ & $+41: 01: 54.0$ & $1 ! 34 \mathrm{E}, 14 ! 24 \mathrm{~S}$ & Kasliwal et al. (2008f) \\
\hline P60-M31OT-080913 (2008-09a) & NGC 224 & 2008 Sep 13.18 & $00: 41: 46.72$ & $+41: 07: 52.1$ & $10^{\prime} .8 \mathrm{~W}, 8^{\prime} .3 \mathrm{~S}$ & Kasliwal et al. (2008e) \\
\hline P60-NGC 891OT-080813 & NGC 891 & 2008 Aug 13.45 & $02: 22: 32.70$ & $+42: 21: 56.1$ & 8’'W, 59”N & Kasliwal et al. (2008c) \\
\hline P60-M31OT-080723 (2008-07b) & NGC 224 & 2008 Jul 23.33 & $00: 43: 27.28$ & $+41: 10: 03.3$ & $8.1 \mathrm{E}, 6.1 \mathrm{~S}$ & Kasliwal et al. (2008d) \\
\hline P60-M82OT-080429 & NGC 3034 & 2008 Apr 29.24 & $09: 55: 21.00$ & $+69: 39: 42.0$ & $165 ” \mathrm{~W}, 64 ” S$ & Kasliwal et al. (2008h) \\
\hline P60-M81OT-071213 & NGC 3031 & 2007 Dec 13.40 & $09: 55: 25.98$ & $+69: 04: 34.8$ & $40 ” \mathrm{~W}, 40 ” \mathrm{~N}$ & Kasliwal et al. (2007) \\
\hline
\end{tabular}

Table 3

Additional M31 Novae

\begin{tabular}{lccl}
\hline \hline Nova Name & Discovery Date & Classification & \\
\hline $2007-10 \mathrm{a}$ & 54380.606 & Fe II & Pietsch et al. (2007); Gal-Yam \& Quimby (2007) \\
$2007-11 \mathrm{f}$ & 54433.716 & $\ldots$ & Ovcharov et al. (2007) \\
$2007-12 \mathrm{~b}$ & 54444.528 & $\mathrm{He} / \mathrm{N}$ & Nakamo; Hornoch; Lee et al. (2007); Bode et al. (2009) \\
$2008-08 \mathrm{c}$ & 54708.127 & Fe II & Valcheva et al. (2008); Hornoch; Di Mille et al. (2010) \\
$2008-10 \mathrm{~b}$ & 54759.698 & Fe II & Henze et al. (2008); Di Mille et al. (2008); Barsukova et al. (2008) \\
$2008-11 \mathrm{a}$ & 54774.438 & Hybrid & Nishiyama; Hornoch; Shafter et al. (2008) \\
\hline
\end{tabular}



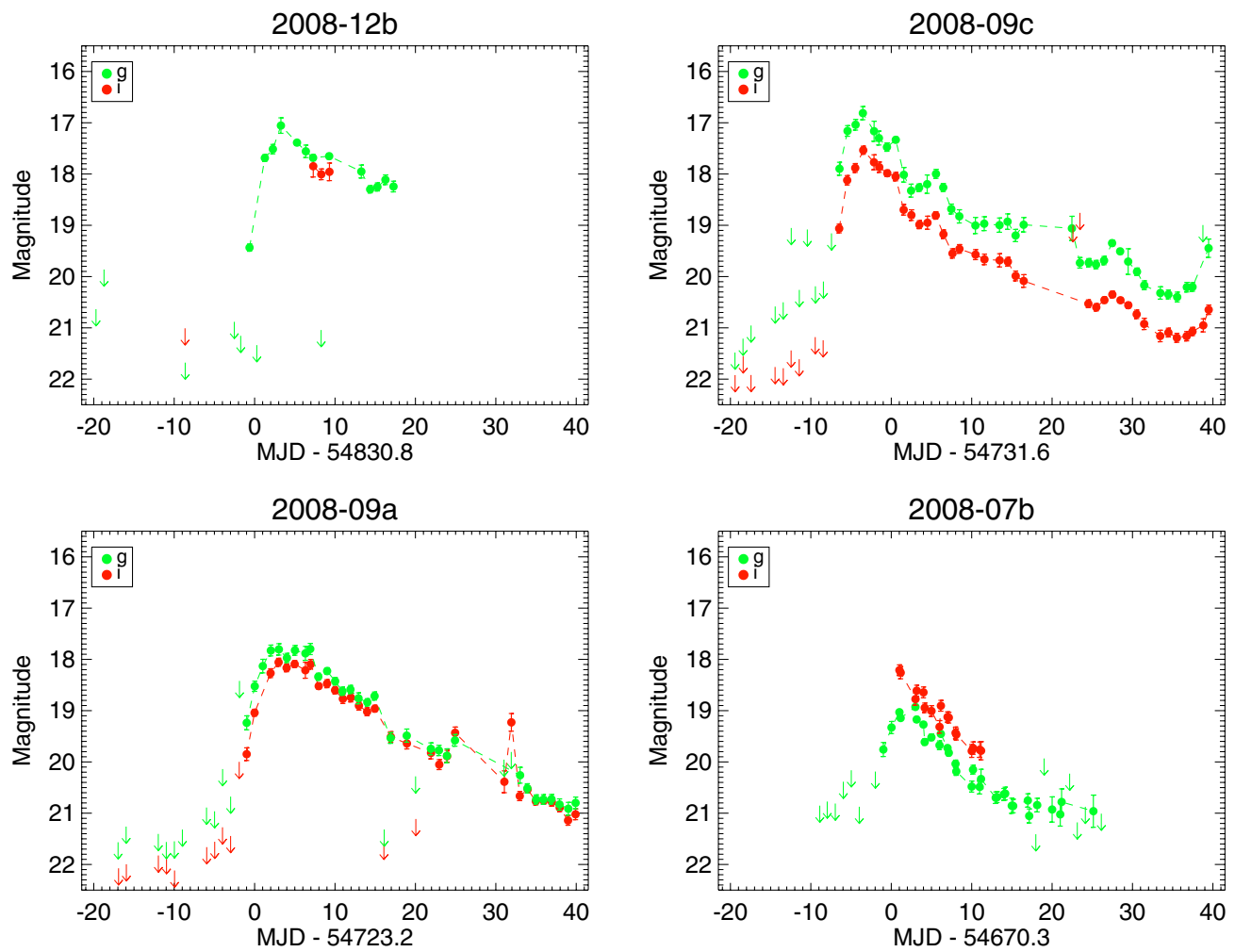

Figure 5. Light curves of novae in M31 discovered by P60-FasTING. Note the well-sampled rise.

(A color version of this figure is available in the online journal.)

Table 4

Spectroscopy

\begin{tabular}{llllcc}
\hline \hline Transient Name & Spectroscopy Date & Telescope & \multicolumn{1}{c}{ Instrument } & Classification & Observer \\
\hline P60-NGC 2403-090314 & 2009 Mar 20.145 & P200 & DBSP (Oke \& Gunn 1982) & Fe Class? & Kasliwal, Ellis \\
P60-M81-090213 & 2009 Feb 18.510 & Keck I & LRIS (Oke et al. 1995) & Fe Class & Ofek \\
P60-M81-081229 & 2008 Dec 31.40 & P200 & DBSP (Oke \& Gunn 1982) & Fe Class & Rau, Salvato \\
P60-M31-081230 & 2008 Dec 31.104 & P200 & DBSP (Oke \& Gunn 1982) & Fe Class & Rau, Salvato \\
P60-M81-081203 & 2008 Dec 4/5,16 & P200, Gemini & DBSP, GMOS-N (Hook et al. 2004) & Fe Class & Kasliwal \\
P60-M81-080925 & 2008 Sep 29.51 & P200 & DBSP (Oke \& Gunn 1982) & Fe Class & Quimby \\
P60-M31-080915 & 2008 Sep 20.2 & HET & LRS (Hill et al. 1998) & Fe Class & Shafter \\
P60-M31-080913 & 2008 Sep 22.4 & HET & LRS (Hill et al. 1998) & Fe Class & Shafter \\
P60-M31-080723 & 2008 Aug 1 & P200 & DBSP (Oke \& Gunn 1982) & Fe Class & Ofek \\
P60-M82-080429 & 2008 May 2.28 & P200 & DBSP (Oke \& Gunn 1982) & Cenko & $\ldots$ \\
P60-M81-071213 & 2007 Dec 15.565 & Keck & LRIS (Oke et al. 1995) & Ofek \\
\hline
\end{tabular}

We emphasize that spectroscopy is crucial in distinguishing between an optical transient which happened to be co-incident with a nearby galaxy and a classical nova. For instance, we took spectra of several optical transient candidates which did not turn out to be novae: a foreground M-dwarf flare in the Milky Way spatially coincident with NGC 7640; a background supernova; a luminous blue variable in NGC 925.

We present light curves of novae in M 31 (Figure 5), M 81 (Figure 6), M 82 (Figure 7), NGC 2403 (Figure 7) and NGC 891 (Figure 9). Additional light curves of novae in M 31 are presented in Figure 8.

Data were reduced in iraf using standard tasks in the NOAO package onedspec and the spectra are shown in Figure 10.

\subsection{X-ray Observations: a Case Study}

Motivated by the fast evolution, we obtained Chandra observations (Director's Discretionary Time; PI: P. Jonker) of P60-M81OT-071213 to explore whether this could be a neutron star or black hole binary event. In a 5.17 ks observation on UT 2007 December 21.779 , we did not detect a single X-ray photon. Thus, 8.4 days after discovery, we derive a $99 \%$ confidence upper limit on the $0.3-7 \mathrm{keV}$ flux of $<9 \times 10^{-4}$ counts $\mathrm{s}^{-1}$. Assuming a power-law spectrum with index of two and column density $\left(n_{\mathrm{H}}\right)$ of $6 \times 10^{20} \mathrm{~cm}^{-2}$, we derive a luminosity limit of $<8.9 \times 10^{36} \mathrm{erg} \mathrm{s}^{-1}$. Extensive X-ray monitoring of classical novae in Andromeda (Henze et al. 2010a, 2010b) suggests that this upper limit is not inconsistent with classical novae.

\section{ANALYSIS}

The primary photometric analysis was to measure the peak absolute magnitude and rate of decline. The peak magnitude had to be corrected for extinction using spectra or colors. The primary spectroscopic analysis was to classify the spectra. 

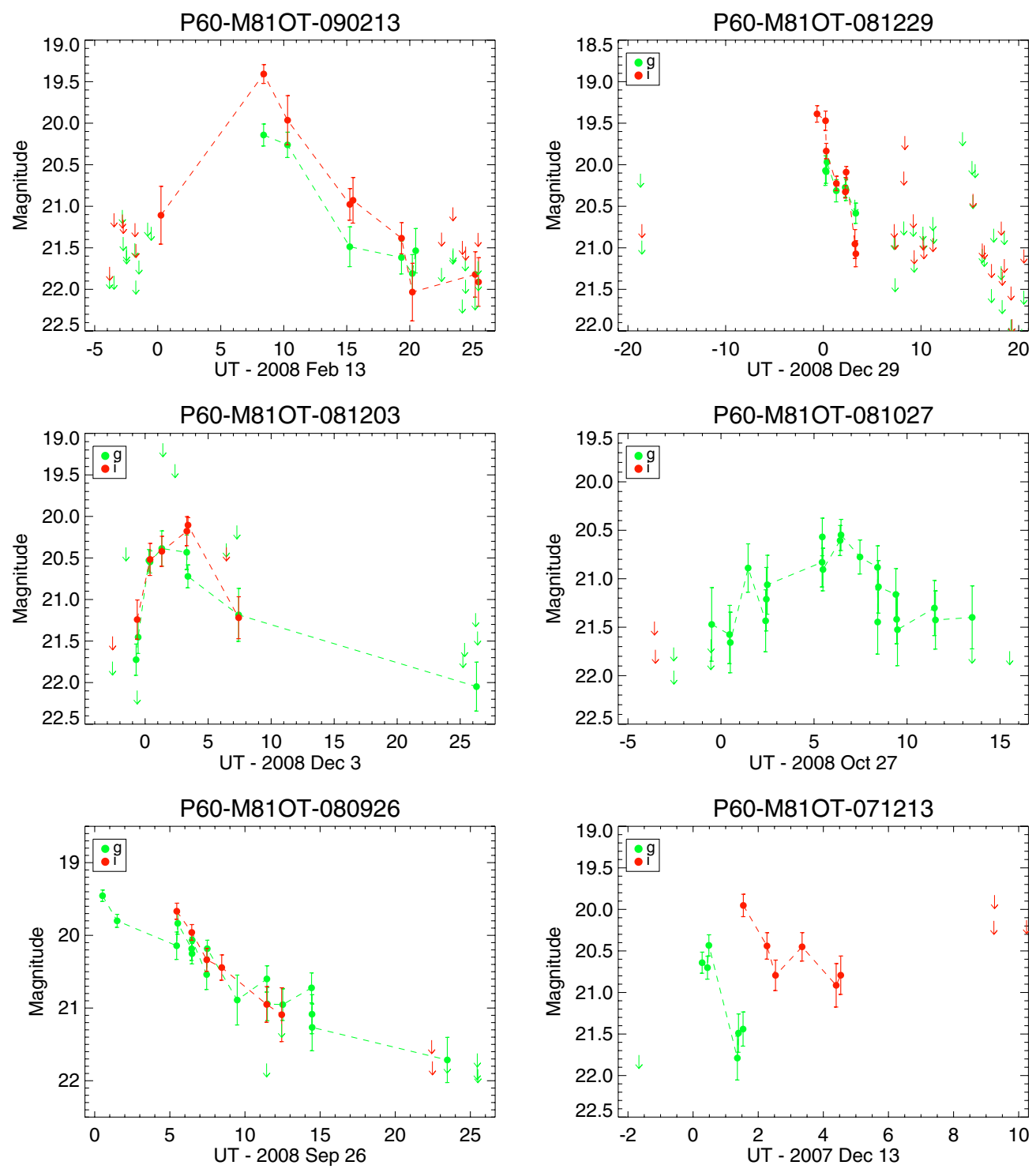

Figure 6. Light curves of novae in M81.

(A color version of this figure is available in the online journal.)

\subsection{Extinction}

A multitude of methods have been used in the literature to measure extinction to novae. Darnley et al. (2006) compared a synthetic dust-free stellar $r-i$ map of M31 to an observed $r-i$ color map of M31 and used the difference between the maps to generate a dust map of M31. The location of the nova on this map determined how much extinction needed to be applied. This assumed that the novae were behind the galaxy and suffered extinction due to the entire column of dust. The average extinction as determined by this method is $A_{i}=0.8$. The galactic extinction along the line of sight of M31 of $A_{i}=0.13$ (Schlegel et al. 1998). Shafter et al. (2009) compared the observed color of the new nova to that of a well-studied nova to derive the extinction. Kogure (1961) used the Balmer decrement $\left(H_{\alpha} / H_{\beta}\right)$ and attributed the excess in the ratio over the theoretical Case B value to dust.

Our preferred method of computing extinction is by using the spectroscopic Balmer decrement where spectra dominated by line emission are available. We subtract the continuum, measure the flux ratio of the two lines, and then use

$$
\begin{aligned}
A_{g} & =3.793 \times E(B-V)=3.650 \times E(g-r) \\
& =3.650 \times \log _{10}\left(H_{\alpha} / H_{\beta}\right)-1.75 \pm 0.14 .
\end{aligned}
$$

The uncertainty comes from the range in expected ratios for Case B of 2.76-3.30.

Our second choice is to use the $g-i$ color of the nova at maximum, compare against the typical $g-i$ color, and attribute the reddening to dust.

van den Bergh \& Younger (1987) compiled photometry of several Galactic novae and derived an average color at maximum of $\langle B-V\rangle_{0}=0.23 \pm 0.06$ mag. Following Shafter et al. (2009), we can use the colors of an A5V star $(T=8200 \mathrm{~K})$ to translate $\langle B-V\rangle_{0}$ to $\langle g-i\rangle_{0}$. Using colors of an A5V star from Kraus \& Hillenbrand (2007), we get

$$
\langle g-i\rangle_{0}=1.88-2.15=-0.27 \mathrm{mag} .
$$



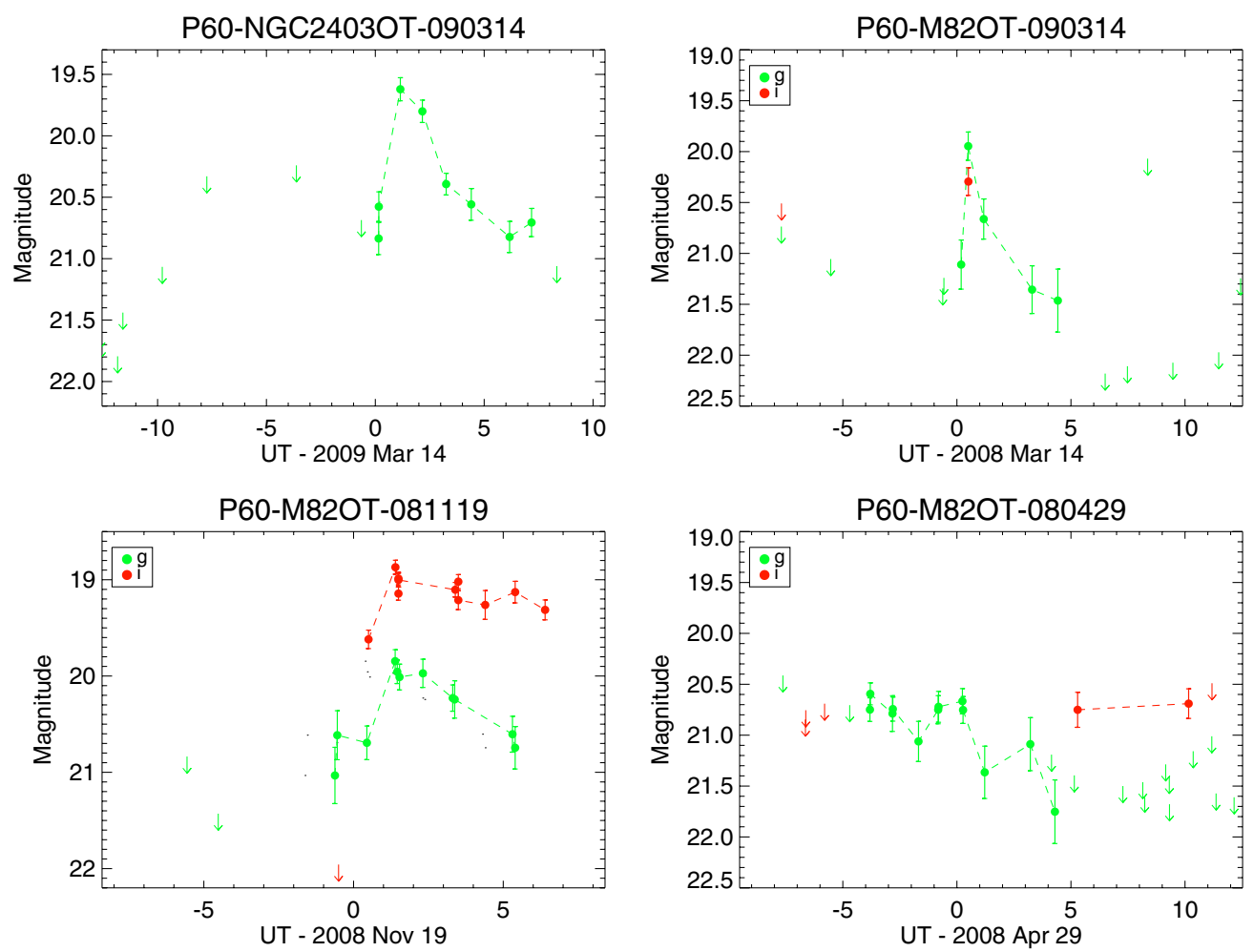

Figure 7. Light curves of novae in M82 and NGC 2403. Note that P60-M82OT-081119 is much redder than typical novae.

(A color version of this figure is available in the online journal.)

Now,

$$
A_{g}=3.793 \times E(B-V)=2.223 \times E(g-i) .
$$

Hence,

$$
\begin{aligned}
A_{g} & =2.223 \times\left[(g-i)_{\mathrm{obs}}-\langle g-i\rangle_{0}\right] \\
& =2.223 \times\left[(g-i)_{\mathrm{obs}}+0.27\right] .
\end{aligned}
$$

For the case of M31N2007-11d (Shafter et al. 2009), where data for both the above options are available, we get consistent answers: $g-i=0.7 \mathrm{mag}$ at maximum suggests $A_{g}=0.75 \mathrm{mag}$ and a Balmer decrement of 4.6 suggests $A_{g}=0.65 \mathrm{mag}$.

If neither a color at maximum nor a spectrum at late-time is available, we use the average line-of-sight extinction to the host galaxy using the Schlegel maps. The uncertainty in extinction calculation significantly contributes to the uncertainty in the peak magnitude.

We note here that for the case of P60-M82OT-081119, the light curve was unusually red for a nova and the extinction correction may be overestimated.

\subsection{Rate of Decline}

The heterogeneity in nova light curves suggests that a single parameter may not characterize the decline well. Traditionally, the time to decay from peak by one magnitude (t1), two magnitudes ( $\mathrm{t} 2$ ), or three magnitudes ( $\mathrm{t} 3$ ) is used. For several novae (e.g., M31N2007-10a, M31N2008-08c, M31N2008-11a), the decline is more or less linear and $\mathrm{t} 1 \mathrm{can}$ be approximated as half of t2. For some novae (e.g., M31N2008-10b), the lightcurve behavior is more complex and this simplification is not applicable. In Table 5, we see that $\mathrm{t} 2$ values (where available) are sometimes larger and sometimes smaller than twice the $\mathrm{t} 1$ value.
Recently, Strope et al. (2010) put together a catalog of very well observed Galactic novae and tried to classify the diversity based on light-curve morphology - they found that $20 \%$ of the novae in their sample have "jitters" or "oscillations" superposed on the decline.

We note here that we did not have data to measure the decline of the nova in NGC 891 and hence it is excluded from further MMRD analysis.

\subsection{Rate of Rise}

Given the cadence of P60-FasTING, we were able to catch several novae on the rise. We define the rate of rise as the average slope between first detection and peak detection and summarize in Table 5. We find a wide range of rise times, from $>1.8 \mathrm{mag} \mathrm{day}^{-1}$ (e.g., M31N2008-11a) to $0.2 \mathrm{mag} \mathrm{day}^{-1}$ (e.g., M31N2008-09a). It is not clear how previous determinations of the MMRD in the literature dealt with the uncertainty in the peak magnitude due to inadequate coverage. In particular, since previous surveys likely had a relatively slower cadence, missing the peak may be a substantial source of error. Slower cadence and/or shallower depth would correspond to a weaker constraint on the rise time of the nova. Due to gaps on account of weather, some of the P60 light curves have constraint weaker than $0.1 \mathrm{mag} \mathrm{day}^{-1}$ on the rate of rise. Hence, we do not use the light curves of P60-M81OT-080926 or P60-M82OT-080429 for subsequent analysis of the MMRD relation.

\subsection{Spectral Classification}

For spectroscopy the primary analysis was to classify the novae by their spectra. The taxonomy of novae were laid out by Payne-Gaposchkin (1964) and McLaughlin (1960). The most prominent feature in all classical novae is Balmer emission. Williams (1992) proposed that there is a two-component 

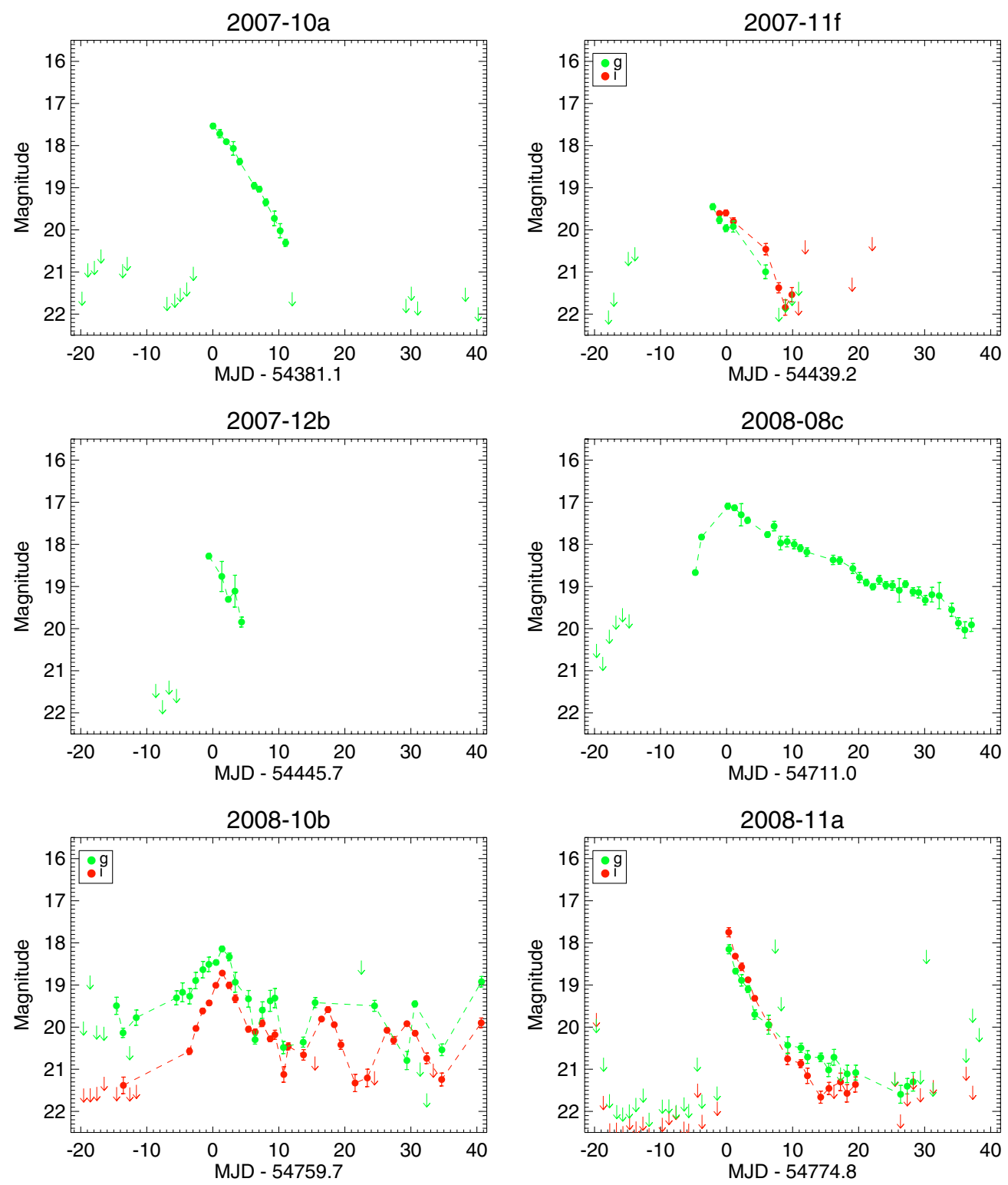

Figure 8. Light curves of additional novae in M31.

(A color version of this figure is available in the online journal.)

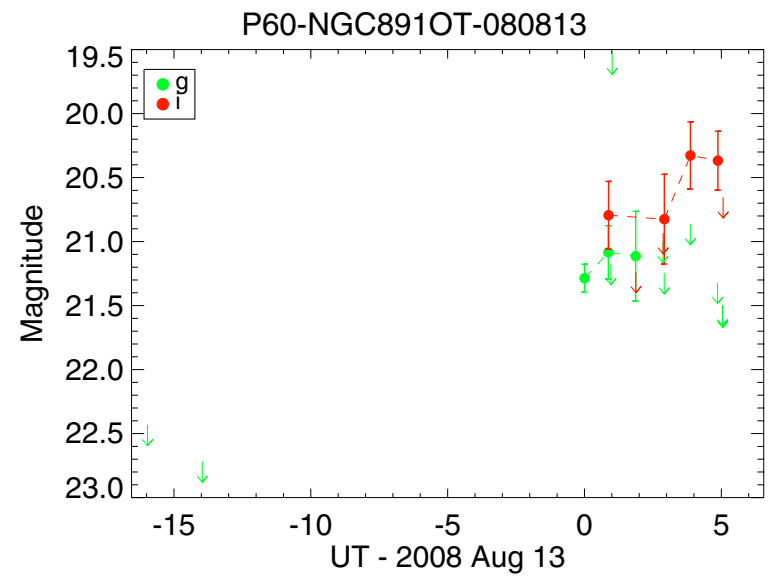

Figure 9. Light curve of possible nova in NGC 891. This was not spectroscopically confirmed and is not used in the MMRD analysis.

(A color version of this figure is available in the online journal.) structure of the emitting gas-discrete shell and continuous wind. If the wind mass-loss rate is low, the effective photosphere is smaller, the radiation temperature higher, the level of ionization of the shell higher, resulting in a shell-dominated $\mathrm{He} /$ $\mathrm{N}$ spectrum. If the wind mass-loss rate is high, it results in a wind-dominated Fe II spectrum.

Thus, classical novae are divided into two principal families-the "Fe" class (dominated by Fe II lines, often low velocity and showing P-Cygni profiles) and "He/N" class (dominated by $\mathrm{He}$ and $\mathrm{N}$ lines, often high velocity and flat or jagged-topped profiles). These evolve into nebular spectra with four classes based on the prominent forbidden lines—standard (e.g., [N II], [O II], [O III]), neon (e.g., [Ne v], [Ne III]), coronal (e.g., [Fe X]), or no forbidden lines. The Fe class novae are expected to evolve into standard or neon nebular spectra. The He/N class are expected to evolve into neon, coronal, or no forbidden line spectra. Some novae are classified as "hybrid" as they start out with high 
Table 5

Nova Characteristics

\begin{tabular}{|c|c|c|c|c|c|c|c|}
\hline Nova Name & $\begin{array}{c}\text { Balmer Decrement } \\
\text { FH } \alpha / \mathrm{H} \beta\end{array}$ & $\begin{array}{c}\text { Color at Peak } \\
g-i(\mathrm{mag})\end{array}$ & $\begin{array}{l}\text { Extinction } \\
A_{g} \text { (mag) }\end{array}$ & $\begin{array}{l}\text { Rate of Rise } \\
\left(\text { mag day }^{-1}\right)\end{array}$ & $\begin{array}{l}\text { Abs-Mag } \\
M_{g} \text { (mag) }\end{array}$ & $\begin{array}{c}\mathrm{t} 1 \\
\text { (days) }\end{array}$ & $\begin{array}{c}\mathrm{t} 2 \\
\text { (days) }\end{array}$ \\
\hline P60-NGC 2403-090314 & 5.0 & $\ldots$ & 0.8 & 1.3 & -9.0 & 3.3 & $>6$ \\
\hline P60-M82-090314 & $\ldots$ & -0.3 & 0.6 & $>1.2$ & -8.5 & $2.4 ?$ & $>3.3$ \\
\hline P60-M81-090213 & $\ldots$ & 0.7 & 2.2 & 0.2 & -9.9 & $5 ?$ & $>10.9$ \\
\hline P60-M31-081230 (2008-12b) & $\ldots$ & -0.17 & 0.24 & 0.6 & -7.5 & 12.3 & \\
\hline P60-M81-081229 & 2.4 & 0.1 & 0.90 & $>0.1$ & -8.7 & 2.9 & 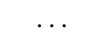 \\
\hline P60-M81-081203 & $\ldots$ & -0.03 & 0.53 & 0.7 & -8.0 & $7.5 ?$ & $>23$ \\
\hline P60-M82-081119 & $\ldots$ & 0.98 & 2.8 & 0.6 & -10.7 & 4.0 & $\ldots$ \\
\hline P60-M81-081027 & $\ldots$ & $\ldots$ & 0.3 & 0.2 & -7.6 & 4.0 & $\ldots$ \\
\hline P60-M81-080926 & 1.4 & -0.2 & 0.13 & $\ldots$ & -8.5 & 8.9 & 14.0 \\
\hline P60-M31-080915 (2008-09c) & 1.4 & -0.72 & 0.24 & 0.4 & -7.8 & 9.1 & 16.6 \\
\hline P60-M31-080913 (2008-09a) & 2.5 & -0.30 & 0.24 & 0.2 & -6.8 & 6.3 & 16.0 \\
\hline P60-M31-080723 (2008-07b) & 14.3 & -0.20 & 2.5 & 0.2 & -7.6 & 5.0 & 12.0 \\
\hline P60-M82-080429 & 6.3 & $\ldots$ & 1.2 & $\ldots$ & -8.5 & 8.1 & $\ldots$ \\
\hline P60-M81OT-071213 & 3.8 & 0.5 & 0.4 & $>0.6$ & -7.8 & 1.0 & $\ldots$ \\
\hline $2007-10 \mathrm{a}$ & $\ldots$ & $\ldots$ & $>0.24$ & $>1.2$ & -7.0 & $4.1 ?$ & 8.6 \\
\hline 2007-11f & $\ldots$ & -0.16 & 0.24 & $>0.1$ & -5.1 & 5.0 & $>8.0$ \\
\hline $2007-12 b$ & $\ldots$ & $\ldots$ & $>0.24$ & $>0.6$ & -6.3 & 3.5 & $>5.0$ \\
\hline $2008-08 c$ & $\ldots$ & $\ldots$ & $>0.24$ & 0.3 & -7.5 & 11.0 & 26.3 \\
\hline $2008-10 b$ & $\ldots$ & -0.58 & $>0.24$ & 0.2 & -6.5 & 6.0 & $12.3 ?$ \\
\hline $2008-11 a$ & $\ldots$ & 0.41 & 1.5 & $>1.8$ & -7.7 & 2.9 & 7.5 \\
\hline
\end{tabular}

velocity Fe II features and quickly evolve into showing $\mathrm{He} / \mathrm{N}$ features (e.g., V745 Sco, V3890 Sgr, M31N2008-11a).

Majority of the P60-FasTING spectra show clear permitted lines from Fe II $(42), \mathrm{Fe}$ II $(37,38)$ and $\mathrm{O}$ I. The line velocities are low, and typical Gaussian FWHM are $<2500 \mathrm{~km} \mathrm{~s}^{-1}$ with the exception of P60-M81-080925 where $\mathrm{H}_{\alpha}$ velocity is $3000 \mathrm{~km} \mathrm{~s}^{-1}$. P60-NGC 2403-090314 shows weak Fe II(42) and weak P-Cygni profiles in Balmer lines and can tentatively also be classified as Fe II class. P60-M81-071213 and P60M82-080429 have very low signal-to-noise ratio $(\mathrm{S} / \mathrm{N})$ and no features other than the Balmer lines are detected; hence, we cannot classify them. Multiple spectra of P60-M81-081203 were taken; initially, the spectra showed a featureless continuum (obtained around maximum light) and later (about 12 days after maximum light), evolved to show Balmer lines, Fe II (42), O I. We summarize spectral classifications in Table 4. For five novae in M31, other groups obtained spectra and we summarize their classification in Table 3.

\section{DISCUSSION}

In comparison to traditional nova searches, P60-FasTING was designed as a faster cadence and deeper survey. Weather permitting, galaxies in the sample were imaged every night to a mean depth of Gunn- $g<21$ mag. Hence, P60-FasTING was sensitive to transients that are less luminous and evolve faster than classical novae.

Given that our light curves are well sampled, and we have spectra or color measurements to correct for extinction, we can securely measure both the maximum magnitude and the rate of decline. To our surprise, as demonstrated in Figure 11, we find that the P60-FasTING nova sample is evidently inconsistent with the MMRD relation (della Valle \& Livio 1995).

In Figure 11, the decay time is measured as the time to decay by one magnitude. To test whether the apparent photometric diversity is consistent with the MMRD over a longer timescale, we plot the time to decay by two magnitudes in Figure 12. Furthermore, we restrict this to the sub-sample of six classical novae in M31 with the best- sampled light curves (see light curves of P60-M31OT-080915, P60-M31OT-080913, P60-M31OT-080723, M31N2007-10a, M31N2008-08c, M31N2008-11a in Figures 5 and 8). Even this sub-sample does not obey the MMRD relation. This scatter is larger than the \pm 0.8 mag predicted on theoretical grounds by Shara (1981).

Despite the atypical photometric signature, the P60-FasTING nova sample shows no spectroscopic peculiarities. In Figure 11, the symbols indicate the spectral class: the majority are Fe II class (stars), a couple are $\mathrm{He} / \mathrm{N}$ class (circles), some have spectra with no prominent features for classification (filled squares), and a few have no spectra (empty squares).

We could hypothesize that some of the P60-FasTING novae are not classical but recurrent (classical novae which recur on timescales shorter than a century) since recurrent novae are also known not to obey the MMRD relation. Recurrent novae are expected to occur in the most massive white dwarfs with high accretion rates. A small amount of mass accreted on a short timescale is sufficient to trigger TNR. Recently, Schaefer (2009) compiled all available photometry over the past century on the 10 recurrent novae in our galaxy, overplotted to compare with the P60-FasTING sample in Figure 13.

There are three ways to test the recurrent nova hypothesis. First, spectra of recurrent novae have high velocities and belong to either He/N or hybrid class (e.g., V3890 Sgr, V745 Sco, V394 CrA in Williams 1992 and Williams et al. 1994). We find that majority of the P60-FasTING novae do not share the spectroscopic properties of recurrent novae. P60-FasTING novae mostly belong to the $\mathrm{Fe}$ II class and have low velocities. Second, recurrent novae are often a few magnitudes brighter than classical novae at quiescence. Schaefer (2009) suggest that recurrent novae range from $-4.1<M_{V}<3.2$ and classical novae range from $1.1<M_{V}<7.0$ at quiescence. Given the distance modulus to these galaxies, this test is within reach of $10 \mathrm{~m}$ class telescopes and easy with $H S T$ (e.g., see Bode et al. 2009). Third, the unambiguous test of whether an eruption is recurrent is to continue to monitor these galaxies for the next several decades until another eruption is witnessed. 


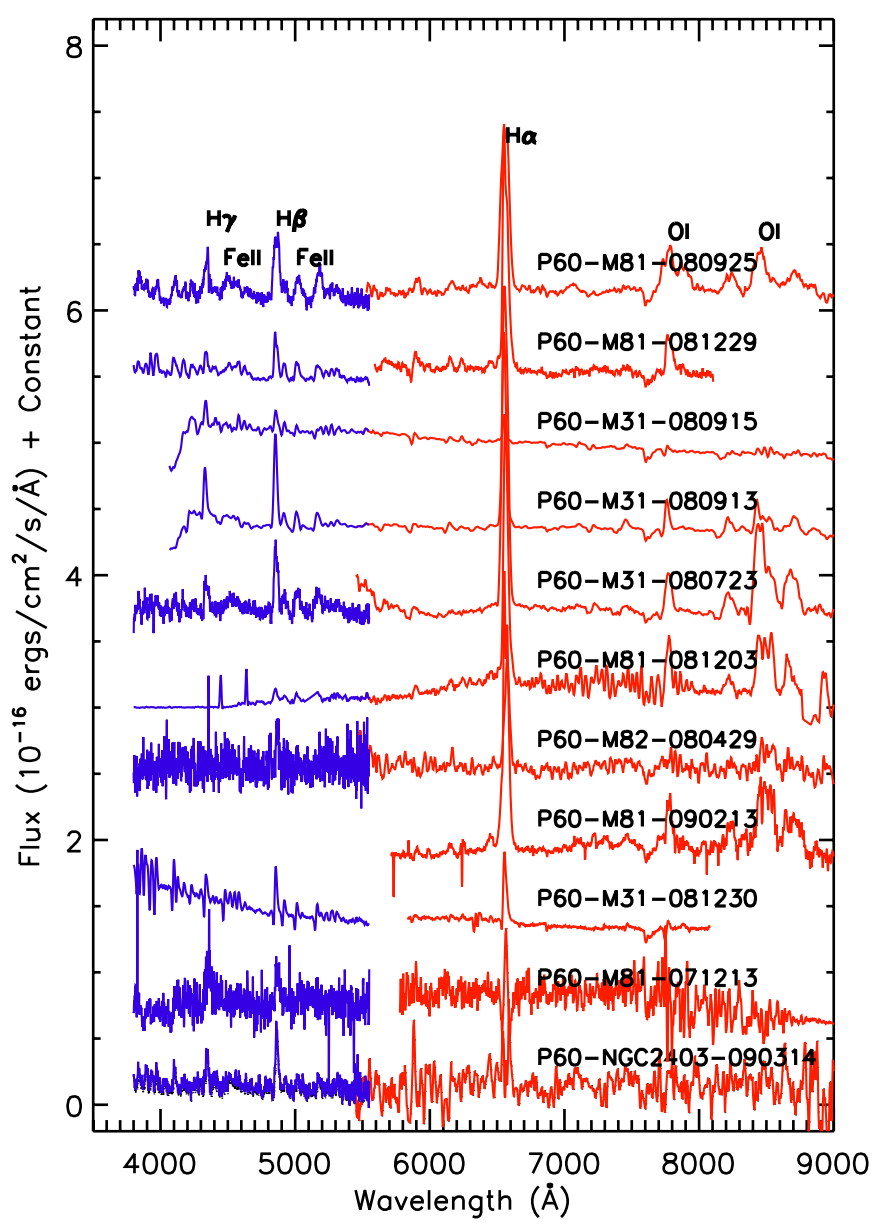

Figure 10. Optical spectra of P60-FasTING novae. The majority are Fe II class. Note that they have been arbitrarily offset along the $y$-axis for clarity.

(A color version of this figure is available in the online journal.)

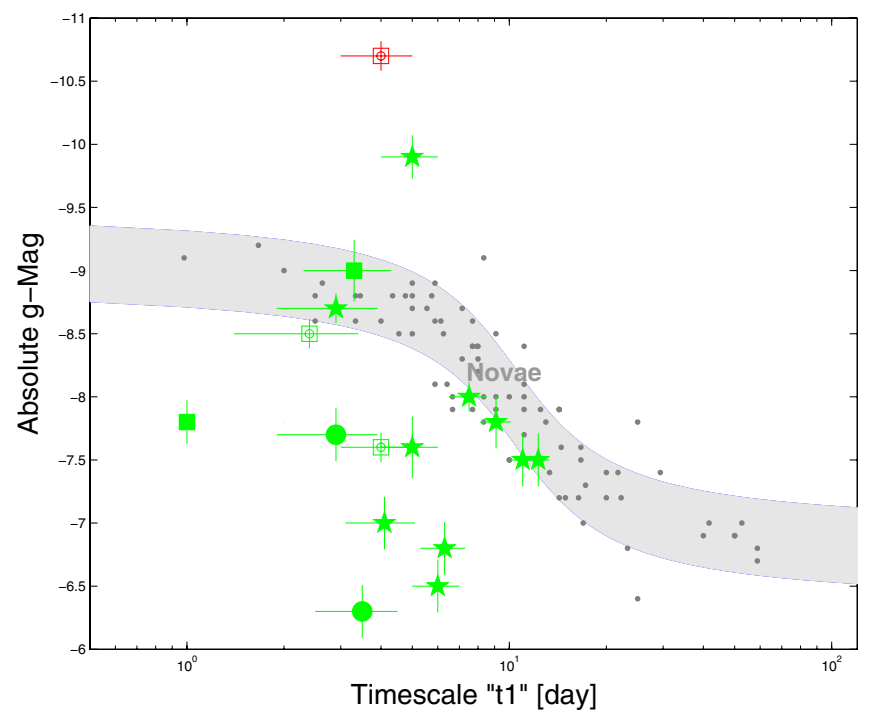

Figure 11. Maximum magnitude (absolute $g$-band) vs. rate of decline (time to decay from peak by $1 \mathrm{mag}$ ). Gray region denotes the della Valle \& Livio (1995) MMRD relation. The dark gray dots denote the nova sample used by della Valle \& Livio (1995) - we derived t 1 by dividing $\mathrm{t} 2$ by two and we converted from $V$-band to $g$-band assuming the colors of an A5V star (Shafter et al. 2009). The P60-FasTING sample is shown with symbols that denote spectral type: $\mathrm{Fe}$ II class (star), $\mathrm{He} / \mathrm{N}$ class (circle), spectrum with no prominent features for classification (squares), and no spectrum (empty square).

(A color version of this figure is available in the online journal.)

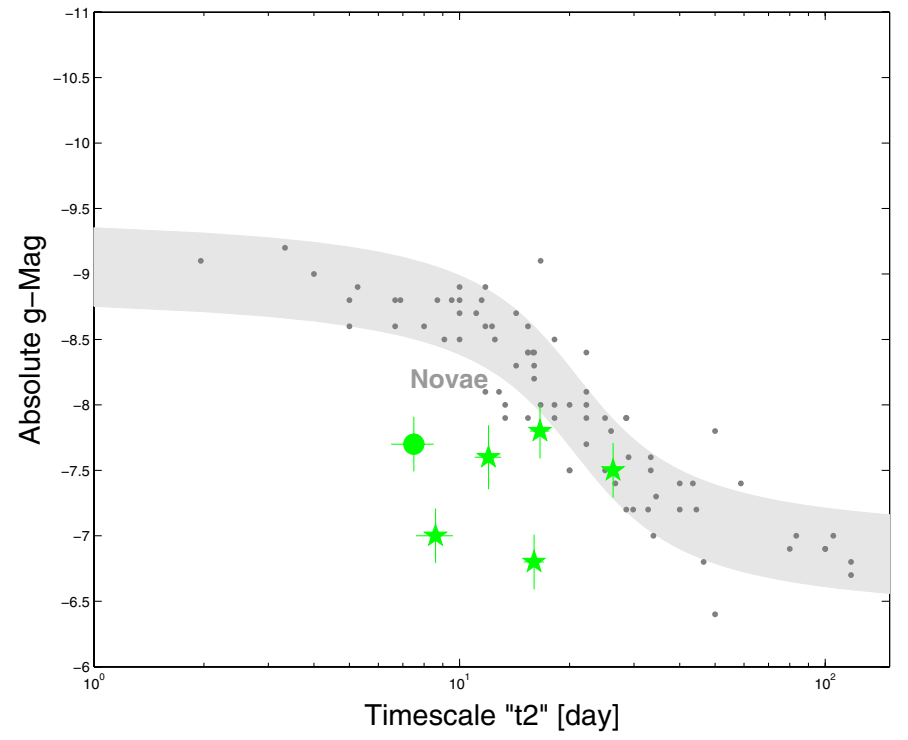

Figure 12. Maximum magnitude (absolute $g$-band) vs. rate of decline (time to decay from peak by $2 \mathrm{mag}$ ). Only the six novae (P60-M31OT-080915, P60M31OT-080913, P60-M31OT-080723, M31N2007-10a, M31N2008-08c, and M31N2008-11a) with the best sampled light curves are shown. Symbols denote spectral type as in Figure 11.

(A color version of this figure is available in the online journal.)

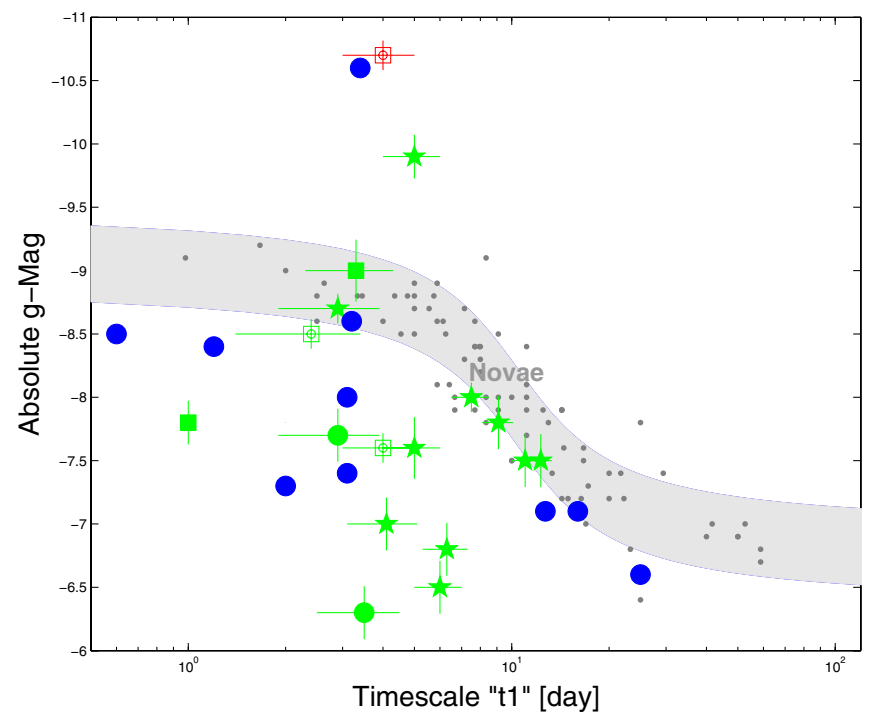

Figure 13. Comparison of the P60-FasTING nova sample (green symbols) with the Galactic recurrent novae (blue circles, data from Schaefer 2009). Symbols denote spectral type as in Figure 11. We note here that there may be a discrepancy in the most luminous recurrent nova (RS Oph), while Schaefer (2009) assume a distance of $4.2 \pm 0.9 \mathrm{kpc}$ and Barry et al. (2008) discuss the merits of different distance measurement techniques and derive a canonical distance of $1.4_{-0.2}^{+0.6} \mathrm{kpc}$. If the latter distance is assumed, the peak luminosity would be -8.3 and not -10.7 .

(A color version of this figure is available in the online journal.)

In order to decipher the nature of this new sub-class of novae, we turn to the fundamental physics of classical novae. The physics is governed by four parameters-mass of the white dwarf, temperature, accretion rate, and composition. The MMRD relation is explained with the mass of the white dwarf being the single, dominant parameter. Could the P60-FasTING sample of faint and fast novae be explained based on an unexplored region of this four-parameter phase space? Could some P60-FasTING novae come from hot and massive 


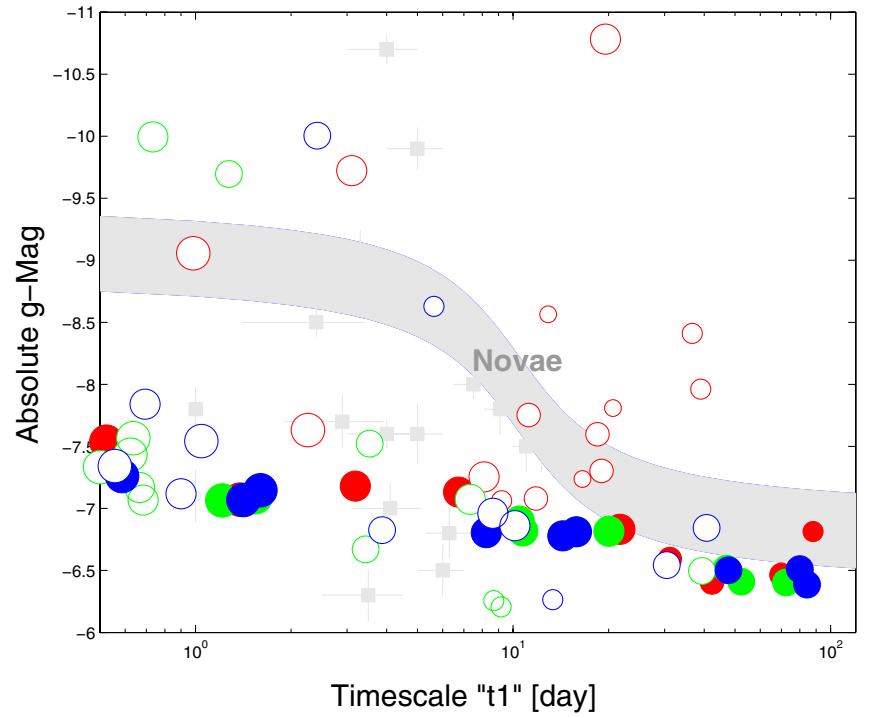

Figure 14. Theoretical results of Yaron et al. (2005) over a wide range of nova parameters-we use the colors of an A5V star to convert L4max to $M_{g}$ and approximate $\mathrm{t} 1$ as $\mathrm{t}_{m l} / 3$. The size of the symbol is proportional to the mass of the white dwarf. The color of the symbol denotes temperature: 10 million $\mathrm{K}$ (red), 30 million $\mathrm{K}$ (green), and 50 million K (blue). Empty circles denote lower accretion rate in the range $10^{-12.3}-10^{-10} M_{\odot} \mathrm{yr}^{-1}$ and filled circles denote higher accretion rate in the range $10^{-9}-10^{-6} M_{\odot} \mathrm{yr}^{-1}$. Note that the density of circles is unrelated to the relative populations.

(A color version of this figure is available in the online journal.)

white dwarfs? If it is hot, then the TNR would not be as explosive and thus, the peak luminosity would be fainter. Also, the higher temperature would result in a smaller amount of envelope mass being sufficient to trigger TNR and thus, the timescale would be faster.

Recent theoretical efforts have explored nova diversity (e.g., Townsley \& Bildsten 2004; Shen \& Bildsten 2009; Epelstain et al. 2007; Jose \& Hernanz 1998; Scott 2000). Yaron et al. (2005) present an extended grid of nova models to explore a wider parameter space (in mass, temperature, and accretion rate) than traditionally explored for classical novae subject to physical constraints (such as conditions for TNR). We summarize the results of the variety of models they run in Figure 14. Some hot and massive white dwarfs with high accretion rates can result in a faint and fast nova population consistent with the P60-FasTING sample. Indeed, Yaron et al. (2005) predict the existence of remarkably small amplitude novae across the entire span of decay rates.

Finally, we note that more than half of the P60-FasTING nova sample is inconsistent with the MMRD. This suggests that faint and fast novae are commonplace and cannot be explained by a rare type of white dwarf.

\section{CONCLUSION}

We conclude that P60-FasTING has uncovered classical novae in a new region in the luminosity-timescale phase space of optical transients. Classical novae span at least two orders of magnitude in time and two orders of magnitude in luminosity. Future surveys would have a large enough sample to meaningfully constrain the relative populations of classical novae in the different areas of phase space.

P60-FasTING was designed as a pilot project, to begin to set the stage for future projects such as Palomar Transient Factory
(PTF ${ }^{9}$; Law et al. 2009; Rau et al. 2009b; Rahmer et al. 2008), PanSTARRS $\left(\mathrm{PS}^{10}{ }^{10}\right.$ ), and Large Synoptic Survey Telescope $\left(\mathrm{LSST}^{11}\right)$. Both PTF and PS1 are now underway. PTF is looking at several nearby galaxies with a similar depth and cadence as P60-FasTING. Among nearby galaxies, PS1 day-cadence fields only cover M31 but are a couple of magnitudes deeper. LSST will be both deeper and faster cadence and cover the visible sky. P60-FasTING is only the trailblazer for the uncovering of a wealth of information about classical novae by near-future synoptic surveys.

We thank Peter Jonker for timely Chandra/DDT observations for the fast evolving nova, P60-M81OT-071213. We thank Marina Orio for discussion, in particular, the suggestion that some of the faint novae may be recurrent novae. We thank A. Shafter, M. Shara, L. Bildsten, and O. Yaron for valuable feedback. We are grateful to A. Becker for making his software hotpants and wcsremap available for public use. M.M.K. thanks the Gordon and Betty Moore Foundation for the Hale Fellowship in support of graduate study. S.B.C. is grateful for generous support from Gary and Cynthia Bengier, the Richard and Rhoda Goldman Fund, and National Science Foundation (NSF) grant AST0908886. M.M.K. thanks the Palomar Observatory staff for their help in maximizing the efficiency and image quality of the Palomar 60 inch. Some of the data presented herein were obtained at the W. M. Keck Observatory, which is operated as a scientific partnership among the California Institute of Technology, the University of California, and the National Aeronautics and Space Administration. The Observatory was made possible by the generous financial support of the W. M. Keck Foundation. The authors recognize and acknowledge the very significant cultural role and reverence that the summit of Mauna Kea has always had within the indigenous Hawaiian community. We are most fortunate to have the opportunity to conduct observations from this mountain.

\section{REFERENCES}

Arp, H. C. 1956, AJ, 61, 15

Barry, R. K., Mukai, K., Sokoloski, J. L., Danchi, W. C., Hachisu, I., Evans, A., Gehrz, R., \& Mikolajewska, J. 2008, in ASP Conf. Ser. 401, ed. A. Evans, M. F. Bode, T. J. O’Brien, \& M. J. Darnley (San Francisco, CA: ASP), 52

Barsukova, E., Fabrika, S., Hornoch, K., Sholukhova, O., \& Valeev, A. 2008, ATel, 1871, 1

Bode, M. F., Darnley, M. J., Shafter, A. W., Page, K. L., Smirnova, O., Anupama, G. C., \& Hilton, T. 2009, ApJ, 705, 1056

Cenko, S. B., et al. 2006, PASP, 118, 1396

Ciardullo, R., Ford, H. C., Neill, J. D., Jacoby, G. H., \& Shafter, A. W. 1987, ApJ, 318,520

Darnley, M. J., et al. 2006, MNRAS, 369, 257

della Valle, M., \& Livio, M. 1995, ApJ, 452, 704

Di Mille, F., Ciroi, S., Orio, M., Rafanelli, P., Bianchini, A., Nelson, T., \& Andreuzzi, G. 2008, ATel, 1818, 1

Di Mille, F., Orio, M., Ciroi, S., Bianchini, A., Rafanelli, P., \& Nelson, T. 2010, Astron. Nachr., 331, 197

Downes, R. A., \& Duerbeck, H. W. 2000, AJ, 120, 2007

Epelstain, N., Yaron, O., Kovetz, A., \& Prialnik, D. 2007, MNRAS, 374, 1449

Ferrarese, L., Côté, P., \& Jordán, A. 2003, ApJ, 599, 1302

Gal-Yam, A., \& Quimby, R. 2007, ATel, 1236, 1

Henze, M., Pietsch, W., Burwitz, V., Hatzidimitriou, D., Reig, P., Primak, N., \& Papamastorakis, G. 2008, ATel, 1790, 1

Henze, M., et al. 2010a, A\&A, 523, A89

Henze, M., et al. 2010b, ArXiv:1010.1461

\footnotetext{
9 http://www.astro.caltech.edu/ptf

${ }^{10} \mathrm{http}: / /$ pan-starrs.ifa.hawaii.edu

$11 \mathrm{http}: / /$ www.lsst.org
} 
Hill, G. J., Nicklas, H. E., MacQueen, P. J., Mitsch, W., Wellem, W., Altmann, W., Wesley, G. L., \& Ray, F. B. 1998, Proc. SPIE, 3355, 433

Hook, I. M., Jørgensen, I., Allington-Smith, J. R., Davies, R. L., Metcalfe, N., Murowinski, R. G., \& Crampton, D. 2004, PASP, 116, 425

Hornoch, K., Scheirich, P., Garnavich, P. M., Hameed, S., \& Thilker, D. A. 2008, A\&A, 492, 301

Hubble, E. P. 1929, ApJ, 69, 103

Jordi, K., Grebel, E. K., \& Ammon, K. 2006, A\&A, 460, 339

Jose, J., \& Hernanz, M. 1998, ApJ, 494, 680

Kasliwal, M. M., Cenko, S. B., Ofek, E. O., Quimby, R., Rau, A., \& Caltech, S. R. K. 2009a, ATel, 1984, 1

Kasliwal, M. M., Cenko, S. B., Ofek, E. O., Quimby, R., Rau, A., \& Kulkarni, S. R. 2009b, ATel, 1934, 1

Kasliwal, M. M., Cenko, S. B., Quimby, R., Rau, A., Ofek, E. O., \& Kulkarni, S. R. 2008a, ATel, 1880, 1

Kasliwal, M. M., Cenko, S. B., Rau, A., Ofek, E. O., Quimby, R., \& Kulkarni, S. R. 2008b, ATel, 1854, 1

Kasliwal, M. M., Cenko, S. B., Rau, A., Ofek, E. O., Quimby, R., \& Kulkarni, S. R. 2008c, ATel, 1663, 1

Kasliwal, M. M., Cenko, S. B., Rau, A., Ofek, E. O., Quimby, R., \& Kulkarni, S. R. 2008d, ATel, 1627, 1

Kasliwal, M. M., Cenko, S. B., Rau, A., Ofek, E. O., Quimby, R., \& Kulkarni, S. R. 2008e, ATel, 1717, 1

Kasliwal, M. M., Cenko, S. B., Rau, A., Ofek, E. O., Quimby, R., \& Kulkarni, S. R. 2008f, ATel, 1719, 1

Kasliwal, M. M., Cenko, S. B., Rau, A., Ofek, E. O., Quimby, R., \& Kulkarni, S. R. 2008g, ATel, 1826, 1

Kasliwal, M. M., Ofek, E. O., Rau, A., Cenko, S. B., Quimby, R., \& Kulkarni, S. R. 2008h, ATel, 1646, 1

Kasliwal, M. M., Quimby, R., Cenko, S. B., Rau, A., Ofek, E. O., \& Kulkarni, S. R. 2008i, ATel, 1764, 1

Kasliwal, M. M., Quimby, R., Rau, A., Ofek, E., Cenko, B., \& Kulkarni, S. 2007, ATel, 1330, 1

Kasliwal, M. M., Rau, A., Salvato, M., Cenko, S. B., Ofek, E. O., Quimby, R., \& Kulkarni, S. R. 2009c, ATel, 1886, 1

Kogure, T. 1961, PASJ, 13, 335

Kraus, A. L., \& Hillenbrand, L. A. 2007, AJ, 134, 2340
Law, N. M., et al. 2009, PASP, 121, 1395

Lee, C., Ries, C., Riffeser, A., \& Seitz, S. 2007, ATel, 1324, 1

Livio, M. 1992, ApJ, 393, 516

McLaughlin, D. B. 1960, in Stellar Atmospheres, ed. J. L. Greenstein (Chicago, IL: Univ. Chicago Press), 585

Oke, J. B., \& Gunn, J. E. 1982, PASP, 94, 586

Oke, J. B., et al. 1995, PASP, 107, 375

Ovcharov, E., Valcheva, A., Kostov, A., Nikolov, Y., Georgiev, T., \& Nedialkov, P. 2007, ATel, 1312, 1

Payne-Gaposchkin, C. 1964, in The Galactic Novae, ed. C. H. P. Gaposchkin (New York: Dover)

Pietsch, W., Burwitz, V., Stoss, R., Updike, A., Hartmann, D., Milne, P., \& Williams, G. 2007, ATel, 1230, 1

Rahmer, G., Smith, R., Velur, V., Hale, D., Law, N., Bui, K., Petrie, H., \& Dekany, R. 2008, Proc. SPIE, 7014, 70144Y

Rau, A., Kasliwal, M. M., \& Salvato, M. 2009a, ATel, 1887, 1

Rau, A., et al. 2009b, PASP, 121, 1334

Schaefer, B. E. 2009, ArXiv:0912.4426

Schlegel, D. J., Finkbeiner, D. P., \& Davis, M. 1998, ApJ, 500, 525

Scott, A. D. 2000, MNRAS, 313, 775

Shafter, A. W., Ciardullo, R., Bode, M. F., Darnley, M. J., \& Misselt, K. A. 2008, ATel, 1832, 1

Shafter, A. W., \& Irby, B. K. 2001, ApJ, 563, 749

Shafter, A. W., Rau, A., Quimby, R. M., Kasliwal, M. M., Bode, M. F., Darnley, M. J., \& Misselt, K. A. 2009, ApJ, 690, 1148

Shara, M. M. 1981, ApJ, 243, 926

Shen, K. J., \& Bildsten, L. 2009, ApJ, 692, 324

Strope, R. J., Schaefer, B. E., \& Henden, A. A. 2010, AJ, 140, 34

Townsley, D. M., \& Bildsten, L. 2004, ApJ, 600, 390

Valcheva, A., Ovcharov, E., Latev, G., Kostov, A., Nikolov, Y., Georgiev, T., \& Nedialkov, P. 2008, ATel, 1687, 1

van den Bergh, S., \& Younger, P. F. 1987, A\&AS, 70, 125

Williams, R. E. 1992, AJ, 104, 725

Williams, R. E., Phillips, M. M., \& Hamuy, M. 1994, ApJS, 90, 297

Yaron, O., Prialnik, D., Shara, M. M., \& Kovetz, A. 2005, ApJ, 623, 398

Zwicky, F. 1936, PASP, 48, 191 